\title{
Combinação de tarefas do enfermeiro e de outros profissionais na atenção primária em saúde: uma revisão sistemática
}

\author{
Silvia Helena De Bortoli Cassiani', Maria Neyrian de Fátima Fernandes², Ludovic Reveiz", \\ José Rodrigues Freire Filho ${ }^{3}$, Fernando Antônio Menezes da Silva ${ }^{1}$
}

Como citar Cassiani SHB, Fernandes MNF, Reveiz L, Filho JRF, Silva FAM. Combinação de tarefas do enfermeiro e de outros profissionais na atenção primária em saúde: uma revisão sistemática. Rev Panam Salud Publica. 2020;44:e82. https://doi.org/10.26633/ RPSP.2020.82

RESUMO

Objetivo. Avaliar a efetividade da estratégia de combinação de tarefas dos profissionais de saúde, e do enfermeiro na Atenção Primária de Saúde.

Método. Revisão sistemática da literatura alinhada com as recomendações do protocolo dos Principais Itens para Relatar Revisões Sistemáticas e Metanálises, incluindo estudos observacionais e de intervenções, sem estabelecimento de período de publicação, independentemente do país de origem e publicados nos idiomas espanhol, inglês e português. As buscas ocorreram nas bases de dados eletrônicas da MEDLINE, CINAHL, LILACS, EMBASE e Web of Science.

Resultados. Incluídos 18 estudos, dos quais 6 foram desenvolvidos nos Estados Unidos; 3 na Holanda; 2 no Reino Unido; um na África do Sul, Austrália, Coreia do Sul, Escócia, Haiti e Japão; e um estudo com desenho observacional prospectivo em 8 países. Os estudos relataram que, a combinação de tarefas entre os profissionais de saúde, possibilita melhor aproveitamento de recursos materiais, otimização do tempo gasto no atendimento, retenção do paciente no tratamento, melhora na qualidade do trabalho com diminuição dos níveis de sofrimento psicológico do enfermeiro e comprometimento e satisfação com o trabalho.

Conclusão. A expansão de tarefas do enfermeiro, assim como de outras categorias de profissionais da saúde, a combinação de tarefas, bem como o desenvolvimento de equipes interprofissionais, são estratégias que podem ser empregadas em situações de escassez de recursos humanos e de má distribuição em áreas urbanas e não urbanas com resultados positivos na melhoria da atenção em saúde da população e satisfação dos profissionais e clientes.

Palavras-chave Atenção primária à saúde; equipe de enfermagem; mão de obra em saúde; recursos humanos; Américas

A possibilidade de os países proverem um serviço de saúde à população que seja adequado, de qualidade e acessível, sem impor riscos financeiros aos indivíduos, é atualmente uma das metas globais (1). A qualidade dos serviços de saúde depende da quantidade, dos conhecimentos e habilidades e do comprometimento da força de trabalho, reconhecida como o componente mais importante e o sustentáculo de qualquer sistema de saúde $(2-4)$.

A promoção do acesso e da cobertura universal de saúde tem relação direta com a adoção de estratégias inovadoras, principalmente nas políticas de recursos humanos em saúde (RHS), que encorajem a eficácia e a eficiência da força de trabalho (5).

\footnotetext{
1 Organização Pan-Americana da Saúde, Washington, D.C., Estados Unidos de América $\bowtie$ Silvia Cassiani, cassianis@paho.org
} 
Assim, para assegurar o acesso, a disponibilidade de infraestrutura adequada e recursos materiais, são necessários o dimensionamento e a distribuição adequada de profissionais. Para a qualidade da atenção, é importante destacar as competências, as habilidades e a maneira como esses profissionais são empregados pelo sistema (5).

Provavelmente, um dos maiores desafios para a promoção do acesso e cobertura universal esteja relacionado às disparidades sociais e econômicas dos países, principalmente aqueles em desenvolvimento (6). Nesses países, a população das áreas rurais e de algumas áreas urbanas, tem dificuldade de acessar os serviços de saúde. A má distribuição, carência de profissionais qualificados e barreiras geográficas e econômicas são os maiores impeditivos para prover a Atenção Primária de Saúde (APS) em algumas regiões (6).

Em áreas remotas, ou mesmo em determinadas áreas urbanas, muitas vezes, o enfermeiro pode ser o único profissional presente com competências para atender a população, implementar terapêuticas ou referenciar o paciente para serviços de maior densidade tecnológica e laboratorial.

A dificuldade de acesso aos médicos, por exemplo, tem levado alguns países a criar programas prevendo atuação de médicos estrangeiros, de estudantes do último ano de graduação ou incentivando financeiramente, ou de outro modo, os profissionais a atuarem naquelas regiões. O Programa Mais Médicos, que funcionou no Brasil de 2013 a 2017 e posteriormente o Programa Médicos pelo Brasil, a partir de 2019, são exemplos de ações do governo brasileiro para aumentar o acesso da população aos médicos.

A inserção de novas tarefas aos profissionais de saúde ou expansão dos papéis dos enfermeiros são alternativas encontradas por mais de $50 \%$ dos países do mundo para aumentar o acesso da população aos profissionais (7). A expansão de papeis, com enfermeiros de prática avançada (EPA), que são enfermeiros que possuem cursos de pós-graduação e junto da equipe da APS, supervisionado ou não pelo médico, avaliam, diagnosticam e cuidam de pacientes e a combinação de tarefas, na qual o enfermeiro ou outro assume tarefas de outros profissionais, em uma equipe interprofissional, dentro de normas e regulamentos estabelecidos pelo país e educação formal adequada são alternativas possíveis para aumentar o acesso da população aos profissionais de saúde $(8,9)$.

Os serviços da APS constituem cenários ideais para se implementar a combinação de tarefas e a prática colaborativa entre os profissionais. A capacidade de implementar o trabalho interprofissional, as habilidades para o trabalho em equipe, a educação e capacitação, e a combinação de tarefas são estratégias reconhecidas como formas de melhorar o acesso, a eficácia e a eficiência dos serviços de saúde $(10,11)$.

Tanto a combinação de tarefas quanto a prática colaborativa são apontadas, pela literatura científica, como métodos eficazes para consolidar a ampliação do acesso e proporcionar serviços de qualidade, centrados nas pessoas, na família e na comunidade $(8,11)$. Todavia, ainda são iniciativas pouco utilizadas nos países da América Latina e Caribe, e recentemente promovidas pela Organização Pan-Americana da Saúde / Organização Mundial da Saúde (OPAS/OMS) (5).

Tendo em vista este cenário, este estudo foi norteado pela pergunta: qual a efetividade da combinação de tarefas no primeiro nível de atenção à saúde? Objetivou-se avaliar a efetividade da estratégia de combinação de tarefas dos profissionais de saúde, e da enfermagem na Atenção Primária de Saúde (APS), com base nos achados da literatura e, propor recomendações para sua aplicação na área de RHS. Considerando que 2020 foi nomeado pela OMS como o ano dos profissionais de enfermagem e de obstetrícia (12) e que esses profissionais são, numericamente, a maior força de trabalho em saúde, decidiu-se enfatizar estudos que abordassem esta categoria profissional.

\section{MATERIAIS E MÉTODOS}

A revisão sistemática foi conduzida com base no protocolo dos Principais Itens para Relatar Revisões Sistemáticas e Metanálises (PRISMA), um checklist e um fluxograma que guiam a escrita de revisões sistemáticas (13). A estratégia PICO: Paciente, Intervenção, Comparação e "Outcomes"- Desfecho foi escolhida por permitir a elaboração de questões de pesquisa de naturezas diversas, incluindo aquelas resultantes do gerenciamento de RHS e materiais (14).

O acrônimo PICO orientou a questão do estudo: (P) População/serviços de saúde da APS; (I) Combinação de habilidades da enfermagem; (C) Combinação de habilidades $X$ intervenção tradicional praticada pela enfermagem; $(\mathrm{O})$ Melhoria do serviço: mensurada por desfechos como aproveitamento de recursos financeiros, melhoria da qualidade dos cuidados, maior retenção dos pacientes no tratamento, melhoria do bem-estar mental do enfermeiro, dentre outros (15).

Os critérios para seleção dos estudos seguiram a PICO, na qual a população de interesse foram equipes da APS que adotaram a prática da combinação de habilidades. Os desenhos dos estudos foram as intervenções (quasi-experimentais e experimentais) e observacionais (coorte e caso-controle); sem limite de período; de qualquer país de origem e publicados nos idiomas espanhol, inglês e português com menção à categoria da enfermagem.

Realizou-se a busca em julho de 2019 nas seguintes bases: 1 . Medical Literature Analysis and Retrieval System Online-MEDLINE via Public/Publisher MEDLINE-PubMed; 2. Cumulative Index to Nursing and Allied Health Literature-CINAHL; 3. Literatura Latino-Americana e do Caribe em Ciências da Saúde-LILACS; 4. Excerpta Medica Database (EMBASE) e 5. Web of Science.

Os descritores foram selecionados no MeSH, DECS e Emtree. Como ainda não existe o descritor skill mix, a estratégia utilizada foi buscar sinônimos e palavras-chaves em artigos previamente publicados sobre a temática. As combinações entre os termos utilizaram os conectores booleanos AND e OR. Os principais descritores, palavras-chaves e sinônimos adotados na estratégia de busca foram: skill mix, Primary healthcare e nurses.

Identificaram-se 5820 artigos. Os artigos encontrados foram exportados para o software Rayyan (16), um instrumento destinado às revisões sistemáticas com capacidade de exclusão de publicações duplicadas, armazenamento e gerenciamento de artigos no processo de revisão.

\section{Seleção de dados}

O processo de inclusão e exclusão está apresentado no diagrama de fluxo baseado no PRISMA (Figura 1). Nessa etapa, foram analisados os títulos e os resumos dos artigos coletados, excluindo-se 4561 de resumos que não abordavam a temática estudada. Em situações de divergência, um pesquisador externo foi convidado para decidir pela inclusão ou exclusão 
FIGURA 1. Diagrama da estratégia de busca e resultados

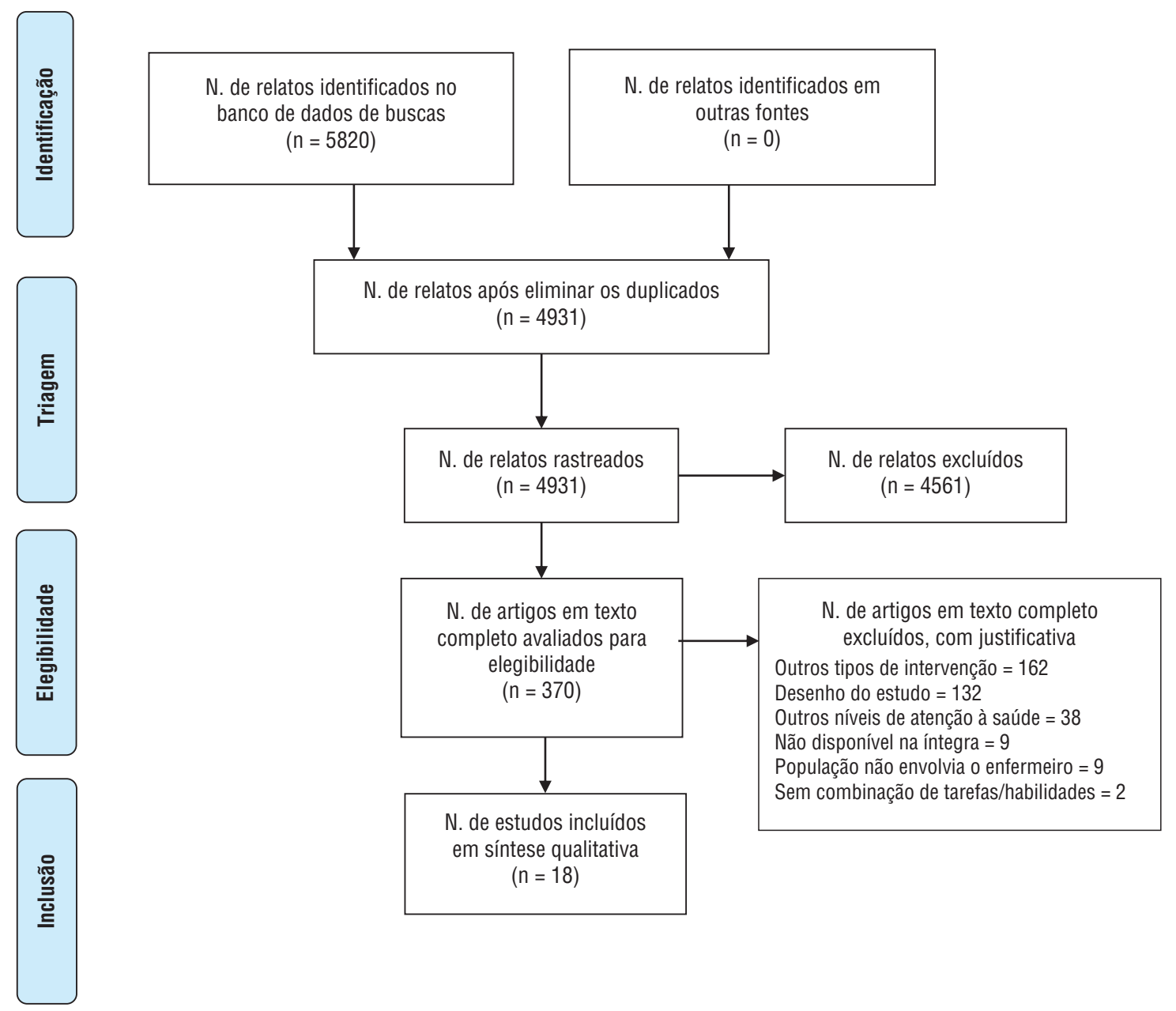

Fonte: preparado pelos autores com base no PRISMA.

do artigo divergente. Em seguida, procedeu-se à leitura integral dos selecionados.

\section{Extração dos dados e avaliação da qualidade}

Um formulário foi criado para analisar os estudos selecionados que incluía: autores e ano de publicação, país, desenho do estudo, objetivo, participantes, amostra, intervenção, controle, desfechos e resultados principais. A otimização de RHS e de recursos financeiros. e a melhoria da qualidade dos cuidados foram os desfechos primários. A permanência dos pacientes no tratamento, a melhoria na saúde mental do enfermeiro, a melhora da qualidade de vida do paciente e o comprometimento com o trabalho foram os desfechos secundários. As intervenções foram classificadas de acordo com a taxonomia da categoria "Profissional que ministra o cuidado e gerenciamento da força de trabalho em saúde" proposta pelo Effective Practice and Organization of Care - EPOC da Cochrane (17).

A qualidade da evidência dos estudos foi avaliada seguindo os critérios recomendados pelo grupo de trabalho GRADE (Grading of Recommendations Assessment, Development and Evaluation) que avalia e classifica em alta, moderada, baixa ou muito baixa a força de recomendação no cuidado em saúde (18). A avaliação de risco de viés foi feita de acordo com o desenho do estudo, seguindo a proposta do EPOC (19) e trabalhadas na interface RStudio do programa estatístico R.

\section{Síntese dos dados}

Realizou-se uma síntese narrativa para integrar os achados reportando o impacto da combinação de tarefas, com o enfoque na equipe de enfermagem. Na primeira etapa, os achados foram descritos de acordo com as suas características. Avaliou-se a heterogeneidade dos artigos. Depois, relatou-se a qualidade metodológica e a descrição da eficácia por comparação das intervenções ou por desfechos de cada estudo.

\section{RESULTADOS}

\section{Descrição dos artigos incluídos}

A estratégia de busca dos estudos e as razões para a exclusão de cada um estão resumidas na Figura 1. Os 18 estudos selecionados, para fazerem parte da análise final, passaram por uma avaliação de qualidade metodológica. Em decorrência da heterogeneidade dos desenhos dos estudos e de sua 
TABELA 1. Características dos estudos incluídos na revisão quanto ao autor, país, participantes, contexto do serviço, grupos de intervenção e de controle ou comparação e intervenção EPOC

\begin{tabular}{|c|c|c|c|c|c|c|}
\hline Autor / Design & País & Participantes (n) & $\begin{array}{l}\text { Contexto do } \\
\text { serviço }\end{array}$ & Grupo intervenção (n) & $\begin{array}{l}\text { Grupo Controle / } \\
\text { comparação (n) }\end{array}$ & Intervenção EPOC \\
\hline $\begin{array}{l}\text { (34) Comparativo } \\
\text { descritivo }\end{array}$ & Escócia & $\begin{array}{l}\text { Profissionais de } \\
\text { Equipes de postos } \\
\text { de saúde (87) }\end{array}$ & $\begin{array}{l}\text { Prevenção } \\
\text { do câncer } \\
\text { cérvico-uterino }\end{array}$ & $\begin{array}{l}\text { Exame por médico, } \\
\text { ou enfermeiros }(20) \text { e } \\
\text { apenas enfermeiro (23) }\end{array}$ & $\begin{array}{l}\text { Exame por médicos } \\
(44)\end{array}$ & $\begin{array}{l}\text { Exame realizado por } \\
\text { médico ou enfermeiro e } \\
\text { outros profissionais }^{* *}\end{array}$ \\
\hline $\begin{array}{l}\text { (20) Comparativo } \\
\text { descritivo }\end{array}$ & EUA & $\begin{array}{l}\text { Lares de idosos } \\
(494)\end{array}$ & $\begin{array}{l}\text { Cuidados para } \\
\text { pessoas idosas }\end{array}$ & $\begin{array}{l}\text { Lares de idosos com } \\
\text { as melhores evolução } \\
\text { clínica (97) }\end{array}$ & $\begin{array}{l}\text { Residências com } \\
\text { as piores evolução } \\
\text { clínica (397) }\end{array}$ & $\begin{array}{l}\text { Combinação de } \\
\text { tarefas na equipe de } \\
\text { enfermagem }^{\star \star}\end{array}$ \\
\hline (21) Experimental & EUA & $\begin{array}{l}\text { Portadores de HIV } \\
\text { (549) }\end{array}$ & $\begin{array}{l}\text { Associação de } \\
\text { Enfermeiras } \\
\text { Visitantes }\end{array}$ & $\begin{array}{l}\text { Clientes de equipes } \\
\text { que implementam a } \\
\text { combinação de tarefas }\end{array}$ & $\begin{array}{l}\text { Pacientes de equipe } \\
\text { tradicional }\end{array}$ & $\begin{array}{l}\text { Modelo de cuidado com } \\
\text { integração de tarefas da } \\
\text { equipe }^{\star \star \star}\end{array}$ \\
\hline (22) Retrospectivo & EUA & $\begin{array}{l}\text { Lares de idosos } \\
\text { (411) }\end{array}$ & $\begin{array}{l}\text { Cuidados para } \\
\text { pessoas idosas }\end{array}$ & $\begin{array}{l}\text { Lares de idosos com } \\
\text { equipes suficientes } \\
\text { (201) }\end{array}$ & $\begin{array}{l}\text { Lares de idosos sem } \\
\text { equipe suficiente } \\
\text { (210) }\end{array}$ & $\begin{array}{l}\text { Combinação de } \\
\text { tarefas na equipe de } \\
\text { enfermagem }^{\star \star}\end{array}$ \\
\hline $\begin{array}{l}\text { (26) Ensaio } \\
\text { clínico } \\
\text { controlado e } \\
\text { randomizado }\end{array}$ & Holanda & $\begin{array}{l}\text { Clientes (1501) } \\
\text { de enfermeiros ou } \\
\text { clínicos gerais }\end{array}$ & Clínica geral & $\begin{array}{l}\text { Pacientes da equipe } \\
\text { com enfermeiro (747) }\end{array}$ & $\begin{array}{l}\text { Pacientes (650) de } \\
\text { clínicos gerais }\end{array}$ & $\begin{array}{l}\text { Combinação de tarefas } \\
\text { entre clínicos gerais e } \\
\text { enfermeiros }^{* *}\end{array}$ \\
\hline $\begin{array}{l}\text { (35) Estudo de } \\
\text { caso }\end{array}$ & Haiti & $\begin{array}{l}\text { Portadores de HIV } \\
(11.114)\end{array}$ & Clínicas & $\begin{array}{l}\text { Modelo baseado nas } \\
\text { tarefas compartilhadas } \\
\text { em equipe }\end{array}$ & Modelo tradicional & $\begin{array}{l}\text { Combinação de } \\
\text { tarefas entre médicos, } \\
\text { enfermeiros e } \\
\text { outros profissionais } \\
\text { comunitários de saúde }\end{array}$ \\
\hline $\begin{array}{l}\text { (31) Estudo } \\
\text { observacional } \\
\text { transversal }\end{array}$ & África do Sul & $\begin{array}{l}\text { Portadores de HIV } \\
\text { (224) }\end{array}$ & $\begin{array}{l}\text { Centro } \\
\text { Comunitário Dia }\end{array}$ & $\begin{array}{l}\text { Modelo de assistência } \\
\text { com Auxiliares de } \\
\text { Farmacêutico e } \\
\text { enfermeiros (164) }\end{array}$ & $\begin{array}{l}\text { Modelo de } \\
\text { atendimento padrão } \\
(60)\end{array}$ & $\begin{array}{l}\text { Modelos de trabalho } \\
\text { com combinação } \\
\text { de tarefas entre } \\
\text { enfermeiros e auxiliares } \\
\text { farmacêuticos }^{\star \star \star}\end{array}$ \\
\hline $\begin{array}{l}\text { (32) ECCR por } \\
\text { clusters }\end{array}$ & Austrália & $\begin{array}{l}\text { Profissionais da } \\
\text { equipe (194) }\end{array}$ & Lares de idosos & $\begin{array}{l}\text { Treinamento e apoio ao } \\
\text { atendimento centrado } \\
\text { na pessoa ( } 45 \text { ) ou } \\
\text { mapeamento da } \\
\text { assistência (56) }\end{array}$ & $\begin{array}{l}\text { Grupo que recebia } \\
\text { cuidados usuais }(23)\end{array}$ & $\begin{array}{l}\text { Modelos de trabalho } \\
\text { com combinação de } \\
\text { tarefas }^{* \star}\end{array}$ \\
\hline $\begin{array}{l}\text { (23) Estudo } \\
\text { correlacional }\end{array}$ & EUA & $\begin{array}{l}\text { Residentes (142) de } \\
\text { Lares de idosos }\end{array}$ & Lares de idosos & $\begin{array}{l}\text { Residentes de lares de } \\
\text { idosos (142) }\end{array}$ & $\begin{array}{l}\text { Correlação } \\
\text { entre hora/dia } \\
\text { por residente, } \\
\text { combinação de } \\
\text { habilidades e taxa de } \\
\text { rotatividade da equipe }\end{array}$ & $\begin{array}{l}\text { Modelos de trabalho } \\
\text { com combinação de } \\
\text { tarefas da equipe de } \\
\text { enfermagem }\end{array}$ \\
\hline $\begin{array}{l}\text { (24) Estudo } \\
\text { comparativo }\end{array}$ & EUA & $\begin{array}{l}\text { Clientes com idade } \\
\geq 65 \text { anos }(4.776)\end{array}$ & Ambulatórios & $\begin{array}{l}\text { Intervenção usando a } \\
\text { Avaliação de Cuidados } \\
\text { de Vulnerabilidade em } \\
\text { clientes no grupo de } \\
\text { intervenção combinada } \\
\text { (2.911) }\end{array}$ & $\begin{array}{l}\text { Grupo controle } \\
\text { agrupado (1.865) }\end{array}$ & $\begin{array}{l}\text { Transferência de tarefas } \\
\text { de médicos para outros } \\
\text { prestadores de cuidados } \\
\text { em saúde }^{* \star}\end{array}$ \\
\hline $\begin{array}{l}\text { (36) Quase- } \\
\text { experimental }\end{array}$ & Japão & $\begin{array}{l}\text { Enfermeiros (174) } \\
\text { de Agência de Saúde } \\
\text { Domiciliar }\end{array}$ & $\begin{array}{l}\text { Agência de } \\
\text { Saúde Domiciliar }\end{array}$ & $\begin{array}{l}\text { Agências de saúde } \\
\text { domiciliar com } \\
\text { combinação de tarefas } \\
\text { entre enfermeiros (44) }\end{array}$ & $\begin{array}{l}\text { Agências de saúde } \\
\text { domiciliar sem } \\
\text { a combinação } \\
\text { de tarefas entre } \\
\text { Enfermeiros (130) }\end{array}$ & $\begin{array}{l}\text { Programa de } \\
\text { combinação de tarefas } \\
\text { para agências de visitas } \\
\text { domiciliares }^{* *}\end{array}$ \\
\hline $\begin{array}{l}\text { (29) Observacional } \\
\text { descritivo }\end{array}$ & Reino Unido & $\begin{array}{l}\text { Clientes na sala de } \\
\text { espera de consulta } \\
(451)\end{array}$ & Sala de espera & Clientes de Enfermeiros & Clientes de Médicos & $\begin{array}{l}\text { Monitoramento rotineiro } \\
\text { da satisfação do cliente } \\
\text { por meio de avaliação da } \\
\text { enfermeira e do médico } \\
\text { clínico geral }^{\star *}\end{array}$ \\
\hline $\begin{array}{l}\text { (30) ECCR por } \\
\text { clusters }\end{array}$ & Reino Unido & $\begin{array}{l}\text { Clientes (16.711) em } \\
\text { consultas }\end{array}$ & Clínica geral & $\begin{array}{l}\text { Triagem telefônica por } \\
\text { clínico geral }(5.001) \text { ou } \\
\text { enfermeiro }(5.255)\end{array}$ & $\begin{array}{l}\text { Consulta presencial } \\
\text { tradicional }(5.138)\end{array}$ & $\begin{array}{l}\text { Modelos de trabalho } \\
\text { com combinação de } \\
\text { tarefas profissional } \\
\text { entre clínicos gerais e } \\
\text { enfermeiros }^{* \star \star}\end{array}$ \\
\hline $\begin{array}{l}\text { (27) Quase- } \\
\text { experimental }\end{array}$ & Holanda & Clientes (12.092) & $\begin{array}{l}\text { Cooperativa de } \\
\text { clínica geral }\end{array}$ & $\begin{array}{l}\text { Clientes de enfermeiros } \\
\text { (987) e de clínicos } \\
\text { geral (5.053). }\end{array}$ & $\begin{array}{l}\text { Clientes (6.052) de } \\
\text { clínicos gerais da } \\
\text { unidade }\end{array}$ & $\begin{array}{l}\text { Assistência provida pela } \\
\text { expansão e combinação } \\
\text { de tarefas de clínicos } \\
\text { gerais e enfermeiros }{ }^{\star \star}\end{array}$ \\
\hline
\end{tabular}


TABELA 1. Características dos estudos incluídos na revisão quanto ao autor, país, participantes, contexto do serviço, grupos de intervenção e de controle ou comparação e intervenção EPOC. (Cont.)

\begin{tabular}{|c|c|c|c|c|c|c|}
\hline Autor / Design & País & Participantes (n) & $\begin{array}{l}\text { Contexto do } \\
\text { serviço }\end{array}$ & Grupo intervenção (n) & $\begin{array}{l}\text { Grupo Controle / } \\
\text { comparação (n) }\end{array}$ & Intervenção EPOC \\
\hline $\begin{array}{l}\text { (28) Quase- } \\
\text { experimental }\end{array}$ & Holanda & $\begin{array}{l}\text { Clínicos geral (162), } \\
\text { enfermeiros (10) e } \\
\text { pacientes (9.503) }\end{array}$ & $\begin{array}{l}\text { Cooperativa de } \\
\text { clínica geral }\end{array}$ & $\begin{array}{l}\text { Equipe } 2 \text { - Clínicos } \\
\text { geral (3), enfermeiro } \\
\text { (1), pacientes (3.166). } \\
\text { Equipe } 3 \text { - Clínicos } \\
\text { geral (2) enfermeiros } \\
\text { (2), pacientes (3.048). }\end{array}$ & $\begin{array}{l}\text { Equipe } 1 \text { - clínico } \\
\text { geral (4) e pacientes } \\
(3.287)\end{array}$ & $\begin{array}{l}\text { Assistência provida por } \\
\text { médico e enfermeiros** }\end{array}$ \\
\hline $\begin{array}{l}\text { (25) Estudo de } \\
\text { coorte }\end{array}$ & EUA & $\begin{array}{l}\text { clientes com } \\
\text { diabetes (368.481) }\end{array}$ & $\begin{array}{l}\text { Instalações de } \\
\text { atendimento } \\
\text { primário para } \\
\text { veteranos (568) }\end{array}$ & $\begin{array}{l}\text { Cuidados primários por } \\
\text { enfermeiros (NPs) a } \\
\text { portadores de diabetes } \\
\text { (67.120) e assistentes } \\
\text { médicos (PAs) a } \\
\text { portadores de diabetes } \\
(25.352) \text {. }\end{array}$ & $\begin{array}{l}\text { Cuidados primários } \\
\text { prestados por } \\
\text { enfermeiros e por } \\
\text { médicos a pessoas } \\
\text { com diabetes } \\
(276.009)\end{array}$ & $\begin{array}{l}\text { Assistência provida por } \\
\text { médicos, auxiliares de } \\
\text { enfermagem/assistentes } \\
\text { de medicina** }\end{array}$ \\
\hline $\begin{array}{l}\text { (37) Observacional } \\
\text { prospectivo }\end{array}$ & $\begin{array}{l}\text { Austrália, Canadá, } \\
\text { Finlândia, Irlanda, } \\
\text { Holanda, Nova } \\
\text { Zelândia, Reino } \\
\text { Unido e EUA. }\end{array}$ & $\begin{array}{l}\text { Multiprofissional } \\
\text { (Médicos/Auxiliares } \\
\text { de enfermagem/ } \\
\text { Assistentes de } \\
\text { medicina) }\end{array}$ & $\begin{array}{l}\text { Força de trabalho } \\
\text { em saúde }\end{array}$ & $\mathrm{N} / \mathrm{A}$ & $\mathrm{N} / \mathrm{A}$ & $\begin{array}{l}\text { Combinação de } \\
\text { tarefas na assistência } \\
\text { médica, auxiliares de } \\
\text { enfermagem/assistentes } \\
\text { médicos** }\end{array}$ \\
\hline $\begin{array}{l}\text { (33) Estudo } \\
\text { comparativo } \\
\text { descritivo }\end{array}$ & Coréia & $\begin{array}{l}\text { Instituições para } \\
\text { idosos registradas } \\
\text { no seguro de } \\
\text { assistência a longo } \\
\text { prazo (1.137) }\end{array}$ & $\begin{array}{l}\text { Instituições de } \\
\text { cuidado a idosos }\end{array}$ & $\begin{array}{l}\text { Instituições de cuidado } \\
\text { de média qualidade } \\
60,2 \% \text { (684), de } \\
\text { primeira classe, } 31,2 \% \\
\text { (355) }\end{array}$ & $\begin{array}{l}\text { Instituições de } \\
\text { cuidado com nível de } \\
\text { qualidade classificado } \\
\text { como baixo (98) }\end{array}$ & $\begin{array}{l}\text { Combinação de tarefas } \\
\text { profissional na equipe } \\
\text { entre as categorias } \\
\text { de enfermagem e } \\
\text { assistentes sociais }\end{array}$ \\
\hline
\end{tabular}

N/A: não se aplica; EPOC, Effective Practice and Organization of Care

Quem ministra o cuidado e como a força de trabalho em saúde é gerenciada / Expansão de função ou mudança de tarefa

Fonte: preparado pelos autores com base em estudos publicados.

TABELA 2. Medidas de desfecho e qualidade da evidência

\begin{tabular}{ll}
\multicolumn{1}{c}{ Medidas de desfecho* } & \multicolumn{1}{c}{$\begin{array}{c}\text { Qualidade da evidência } \\
(\text { GRADE })^{* *}\end{array}$} \\
$\begin{array}{l}\text { Aproveitamento de recursos financeiros (34) } \\
\text { Melhoria da qualidade dos cuidados (20) }\end{array}$ & Muito baixa \\
Maior permanência no tratamento (21) & Baixa \\
$\begin{array}{l}\text { Aproveitamento de recursos financeiros por } \\
\text { redução de dias de internamento (21) }\end{array}$ & Moderada \\
Melhoria da qualidade dos cuidados (22) & Muito baixa \\
Aproveitamento de recursos financeiros (26) & Baixa \\
Aproveitamento de RHS*** (35) & Alta \\
Aproveitamento de recursos financeiros (31) & Baixa \\
Melhoria na saúde mental do enfermeiro (32) & Muito baixa \\
Melhora da qualidade de vida do paciente (23) & Alta \\
Melhoria da qualidade dos cuidados (24) & Baixa \\
Comprometimento no trabalho (36) & Alta \\
Aproveitamento dos RHS (30) & Moderada \\
Aproveitamento de recursos materiais (27) & Moderada \\
Aproveitamento de recursos financeiros (27) & Alta \\
Aproveitamento de RHS (28) & Alta \\
Aproveitamento de RHS (25) & Alta \\
Aproveitamento de RHS (37) & Moderada \\
Melhoria da qualidade dos cuidados (37) & Baixa \\
Melhoria da qualidade dos cuidados (33) & Baixa \\
\hline
\end{tabular}

Alta: é improvável que trabalhos adicionais irão modificar a confiança na estimativa do efeito. Moderada: trabalhos futuros poderão modificar a confiança na estimativa de efeito, podendo, inclusive, modificar a estimativa. Baixa: trabalhos futuros provavelmente terẫo um impacto importante em nossa confiança na estimativa de efeito. Muito baixa: qualquer estimativa de efeito é incerta.

*Um estudo (31) não apresentou desfecho que se relacionasse diretamente com o questionamento da revisão. ${ }^{* *}$ Grading of Recommendations Assessment, Development, and Evaluation

**Recursos Humanos em Saúde

Fonte: preparado pelos autores com base em estudos publicados. heterogeneidade estatística $\left(\mathrm{Q}=2843,15, \mathrm{~T}^{2}=0,03, \mathrm{I}^{2}=99,51\right.$, $\mathrm{P}<0,01)$ não foi possível realizar uma metanálise.

Dos dezoito estudos, seis foram desenvolvidos nos Estados Unidos (EUA) (20-25); três na Holanda (26-28); dois no Reino Unido (29,30); um estudo em cada um dos seguintes países: África do Sul (31), Austrália (32), Coreia do Sul (33), Escócia (34), Haiti (35) e Japão (36); e um estudo (37) com desenho observacional prospectivo em oito países (Austrália, Canadá, EUA, Finlândia, Irlanda, Holanda, Nova Zelândia e Reino Unido) (Tabela 1).

Estes estudos objetivaram analisar a combinação de tarefas dentro da equipe de enfermagem e desta com médicos, assistentes sociais e farmacêuticos. (Tabela 1). Os participantes dos estudos incluíam equipes que: 1 . prestavam cuidados a idosos $(20,22-24,32,33,36) ; 2$. realizavam a consulta inicial na APS (26$30) ; 3$. ministravam cuidados aos portadores de $\operatorname{HIV}(21,31,35)$; 4. realizavam o exame de prevenção do câncer cervical (34); 5. assistiam aos portadores de diabetes (25); e 6. compunham os RHS (37).

\section{Descrição da qualidade metodológica / risco de viés}

As informações metodológicas e a qualidade das evidências estão resumidas nas tabela 1 e tabela 2. Cinco empregaram como desenho o estudo observacional $(22,25,29,31,37)$; outros cinco, o comparativo $(20,23,24,33,34)$; três ensaios clínicos randomizados $(26,30,32)$; três quasi-experimental $(27,28,36)$; um experimental (21); e um empregou o estudo de caso (35). O risco de viés foi variado nos estudos incluídos. As Figuras 2 e 3 fornecem uma visão geral. 
FIGURA 2. Risco de viés representando o percentual de risco de viés dos estudos incluídos

Relato de desfecho seletivo

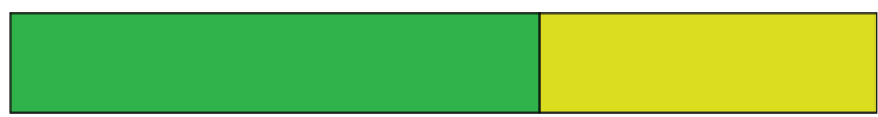

Outras fontes de viés

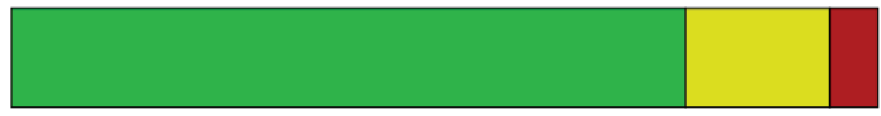

Ocultação de alocação

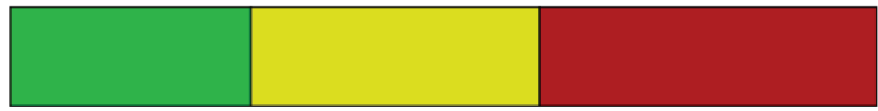

Geração da sequência aleatória

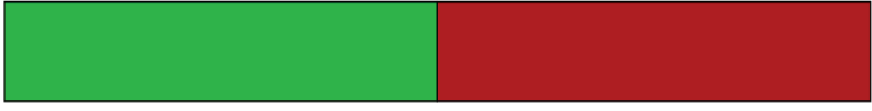

Desfechos incompletos

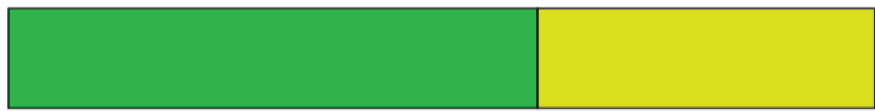

Cegamento de avaliação de desfecho

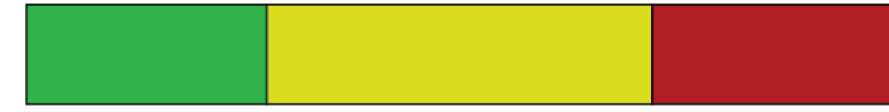

\begin{tabular}{lccc}
\hline $0 \%$ & $25 \%$ & $50 \%$ & $75 \%$ \\
\\
$\square$ Baixo risco de viés & $\square$ Risco de viés incerto & Alto risco de viés \\
\hline
\end{tabular}

Fonte: preparado pelos autores com base em estudos publicados.

\section{Descrição da eficácia das intervenções e desfechos primários}

As intervenções EPOC classificadas de acordo com a categoria "Profissional que ministra o cuidado e gerenciamento da força de trabalho" (17) (Tabela 1). A subcategoria prevalente foi "Expansão de funções ou mudança de tarefa e equipe" (20, 21, 23-25, 29-31, 33-37), relacionada à expansão de tarefas executadas por uma determinada categoria ou mudanças de tarefas de profissional para outro. Na subcategoria "Modelos de equipe" $(22,26-28,32)$ se agruparam intervenções ou estratégias para atingir um nível adequado e uma combinação ideal de profissionais para a prestação de um cuidado, aproximando-as dos pressupostos da prática colaborativa interprofissional (17).

Os desfechos primários (Tabela 2) foram relacionados ao melhor aproveitamento dos recursos financeiros $(21,26-$ $28,31,34)$, tais como as práticas de triagem cervical por enfermeiro ou clínico geral. Nota-se que apresentaram uma relação de custo variando de $£ 0,29$ a 14,67 e deram um retorno de $£ 1,75$ contra $£ 0,45$ para cada $£ 1,0$ investido (34). Foi encontrada uma diferença média significativa nos custos diretos da consulta do enfermeiro comparado aos do clínico geral (26). $\mathrm{O}$ modelo de cuidado interprofissional envolvendo o assistente farmacêutico supervisionado e o enfermeiro quando comparado com o modelo padrão de atendimento que envolve apenas o farmacêutico ministrando os cuidados sobre os antirretrovirais apresentou uma média anual de US $\$ 12,56$ contra US $\$ 128,99$ de custos em comparação com o modelo padrão (31).

As consultas do enfermeiro também foram associadas a menos prescrições de medicamentos e menos referências à emergência do que as consultas com o clínico geral (Risco Relativo-RR $=0,78 ; 95 \%$ Intervalo de Confiança-IC $=0,53-1,15$ ).

A melhoria na qualidade dos cuidados a pacientes na APS foi relatada em quatro estudos $(20,22,25,33)$. O modelo adotado por enfermeiros que realizavam visitas aos domicílios dos pacientes em tratamento de HIV resultou na redução do tempo de internação, um dos principais fatores de custo geral do tratamento (Diferença Média-DM = 54,7; 95\%; IC $=-13,20-122,60)(21)$.

A evolução clínica de idosos em instituições de longa permanência mostrou que a melhoria dos cuidados foi maior naquelas com maiores números de enfermeiros. A relação entre o nível e a combinação da equipe de enfermagem e suas associações com a qualidade do atendimento nestas instituições se mostrou positiva (22). A atuação interprofissional, foi positivamente relacionada à qualidade do atendimento. Instituições de longa permanência com maior número de profissionais de enfermagem foram mais propensas a ser classificadas como superiores na qualidade do atendimento (33).

Adelegação de tarefas a outros profissionais melhorou os indicadores de qualidade de atendimento em situações corriqueiras com pacientes idosos nas práticas comunitárias como quedas, incontinência urinária e demência. A análise dos indicadores de qualidade na delegação de tarefas foi de 0,36 para as realizadas por médicos e 0,55 para EPA ou assistente médico (24).

Com relação a pacientes portadores de diabetes, quando comparado os resultados dos níveis de colesterol, pressão arterial sistêmica e concentração de hemoglobina glicada destes pacientes acompanhados por assistentes de médico, EPA e médicos não foi encontrada variação clinicamente significativa 
FIGURA 3. Resumo do risco de viés de acordo com a avaliação da qualidade metodológica

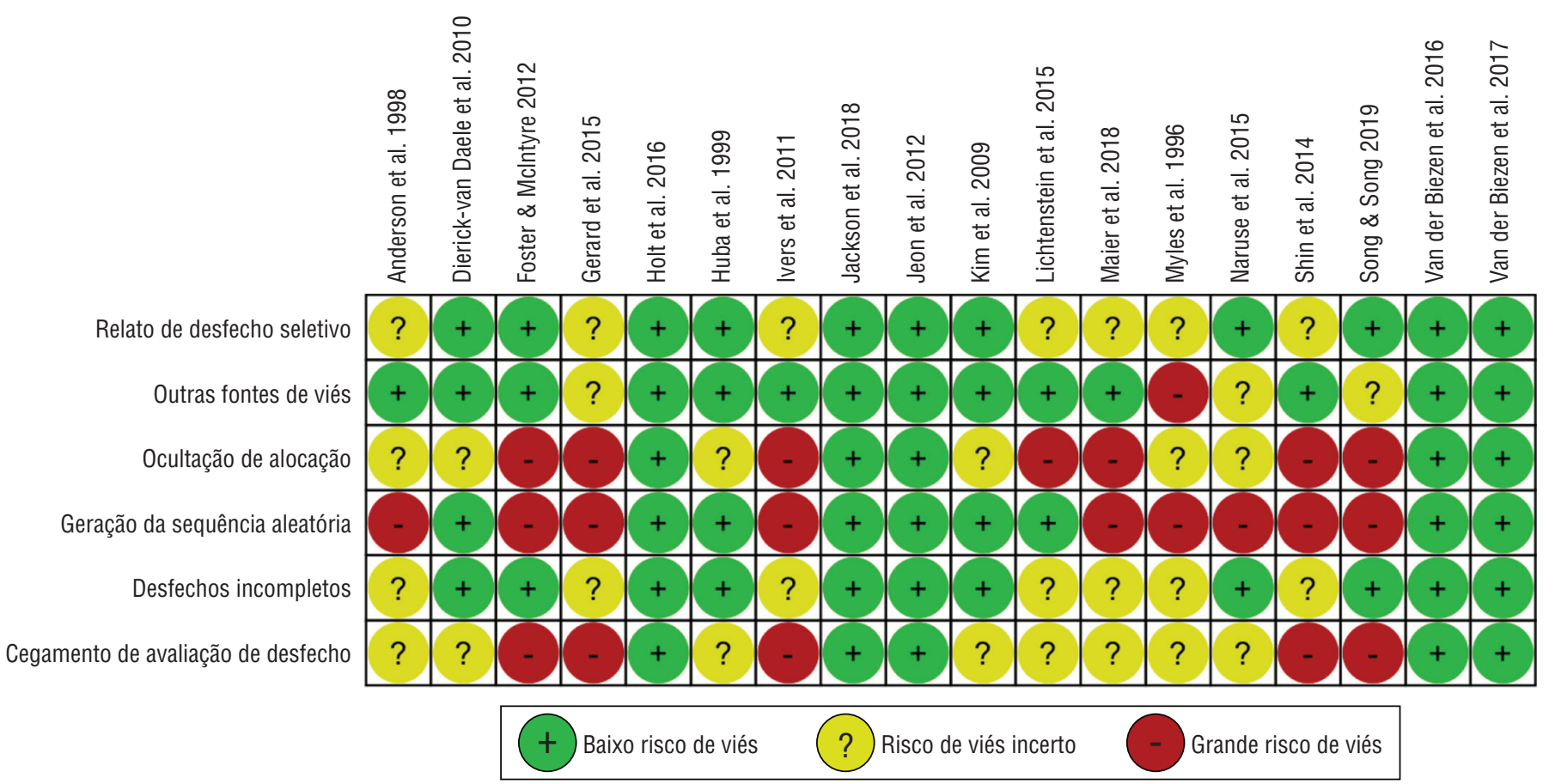

Fonte: preparado pelos autores com base em estudos publicados.

entre os pacientes atendidos pelos três tipos de profissionais. Esse dado sugere que resultados semelhantes, no atendimento de pacientes com doenças crônicas, podem ser alcançados pelos médicos, nurse practitioner e assistentes de médico (25).

Quanto ao desfecho da distribuição adequada de RHS $(28,35,37)$, nota-se que a mudança nas tarefas de médicos para enfermeiros e agentes comunitários de saúde em comparação com o modelo tradicional de atendimento, relacionadas aos cuidados com pacientes com HIV, foi observada em clínicas do Haiti (35). No modelo de combinação de tarefas, apenas $2 \%$ das tarefas eram realizadas exclusivamente por médicos, comparados com $64 \%$ das tarefas no modelo tradicional. No novo modelo, os enfermeiros participaram de uma proporção maior de tarefas, chegando a realizar 92\% das 135 atividades clínicas (35).

Estudo realizado na Holanda evidenciou que o aumento do número de EPAs (28), possibilitou aos clínicos gerais tratarem mais pacientes com problemas de saúde de maior complexidade e evidenciou que equipes de APS, com uma razão de até dois médicos e dois EPAs foram capazes de prestar assistência a todos os pacientes durante um fim de semana (28). Nesse sentido, a combinação de tarefas entre enfermeiros e médicos gerou uma estimativa de redução de 10 a $16 \%$ da carga de trabalho de médicos clínicos gerais em uma década na Holanda (37).

\section{Desfechos secundários}

A combinação de tarefas também beneficiou o próprio profissional de enfermagem, com influência na sua saúde mental, melhorando os níveis de exaustão emocional devido ao trabalho em equipe interdisciplinar, aumentando a qualidade de serviços (32), satisfação e comprometimento com o trabalho (36). Além disto, uma adequada combinação de habilidades propiciou maior permanência do paciente no tratamento por causa da melhora da qualidade do cuidado (21).

Finalmente, ao comparar o planejamento dos RHSs em oito países: Austrália, Canadá, EUA, Finlândia, Irlanda, Holanda, Nova Zelândia e Reino Unido. Esses países introduziram novas profissões da saúde nos serviços, tais como assistente de médico e EPA, ambos com formação avançada, evidenciou que a combinação de habilidade desses profissionais pode reduzir as dificuldades da população causadas pela escassez ou falta de médicos nos serviços de APS (37).

\section{DISCUSSÃO}

A combinação de tarefas nos serviços de saúde e especificamente nos de APS se mostrou positiva tanto para a população atendida como para os profissionais. A combinação de tarefas e a prática colaborativa, em sistemas de saúde de países em desenvolvimento, apontam que esses modelos se apresentam como alternativas na superação das dificuldades impostas pela escassez de profissionais de saúde, sobretudo em áreas de difícil acesso ou de baixa densidade de profissionais (38).

A enfermagem ocupa um papel importante nos serviços, tanto pelo quantitativo da força de trabalho em saúde quanto por sua capacidade de fortalecer os sistemas de saúde (39), sendo um pilar dos serviços de saúde e um fator relevante para o cumprimento das metas de acesso e cobertura universal à saúde, bem como do alcance dos Objetivos de Desenvolvimento Sustentável, o que levou a OMS a dedicar o ano de 2020 para solicitar o investimento neste grupo profissional.

Dos 18 estudos incluídos, apenas oito eram de países da Região das Américas: Canadá, EUA e Haiti. Esses países apresentaram investigações sobre o tema da combinação de tarefas para a 
melhoria dos serviços da APS. Desses países, o Haiti, assim como a maioria dos países da região, enfrenta dificuldades com o baixo número de médicos e enfermeiros nos serviços (40).

A África do Sul (31) enfrenta escassez de profissionais farmacêuticos e apresenta estratégia para melhorar a assistência aos portadores de HIV combinando as tarefas e reforçando a atuação interprofissional entre o enfermeiro e o assistente de farmacêutico (35).

Os países da Região das Américas enfrentam um contexto com déficit de profissionais em determinadas áreas, baixa retenção de profissionais em áreas não urbanas, condições precárias de trabalho e falta de motivação dos profissionais, dentre outros problemas, o que dificultam o processo de expansão dos serviços na APS (5). É nesse contexto que políticas que regulam e investem na combinação de tarefas dos profissionais de saúde: médicos, enfermeiros e farmacêuticos e apoiam o desenvolvimento de equipes interprofissionais podem ser implementadas para melhorar a resolutividade dos serviços (41).

Análise realizada em 50 estados dos EUA, de 2010 a 2016, mostrou que o número de EPA em comunidades com residentes de baixa renda aumentou de 19,8 para 41,1 por 100.000 / habitantes, enquanto a quantidade de médicos se manteve relativamente constante. Em comunidades rurais, esse número aumentou de 25,2 para 41,3 por 100.000 /habitantes, enquanto os médicos diminuíram de 59,5 para 47,8 (42).

A combinação de tarefas e a regulamentação da expansão de tarefas permitem mais autonomia aos enfermeiros e/ou demais profissionais no provimento de cuidados na APS, valorizam a formação educacional e técnica do profissional e realocam o médico ou outro profissional para outros procedimentos mais complexos e especializados. Trata-se de uma "substituição vertical" em que enfermeiros e outros profissionais da saúde assumiriam tarefas que antes eram consideradas exclusiva dos médicos (37). Essas tarefas podem ser executadas de acordo com protocolos, tais como exames, tratamento e gerenciamento de pacientes com doenças crônicas estabelecidas $(37,43)$. Nesse cenário, há evidências de que entre 25 e $75 \%$ das tarefas realizadas por médicos podem ser realizadas por enfermeiros na APS (44).

Os custos em saúde podem ser reduzidos com a implementação da combinação de tarefas e o trabalho interprofissional. Estudo ilustrou o impacto na Holanda do emprego de EPA, ao invés do clínico geral, para realizar consultas de problemas comuns de saúde, implicando em uma redução de $£ 8,21$ em favor da consulta do enfermeiro. Os autores ressaltaram que essa economia pode ser utilizada para redesenhar a APS, no sentido de permitir que o médico realize mais consultas com pacientes portadores de outras condições complexas de saúde (26).

A melhoria na saúde mental de enfermeiros proporcionada pela combinação de tarefas tem relação com a percepção e valorização do próprio conhecimento e está diretamente ligada com a atmosfera positiva do local trabalho criado por um ambiente cooperativo (45). Esses fatores são frutos de uma combinação eficaz.

A OPAS/OMS tem estimulado seus países membros a promover a implementação e formação de EPA como aqueles profissionais com formação de mestrado para prover diagnóstico de doenças agudas leves e crônicas e a enfermeira gestora de casos e EPA especialista em obstetrícia (43). Também estimula a educação interprofissional e a prática colaborativa.
Os resultados encontrados e apresentados nos diversos artigos analisados também abrem espaço para a importância da educação interprofissional (EIP) no processo de formação dos profissionais da saúde. Os temas relacionados à combinação de tarefas, equipes interprofissionais e expansão do papel do enfermeiro têm capacidade para o estabelecimento de trabalho colaborativo, integrado, superação dos estereótipos profissionais, aprendizado compartilhado e avanços para a APS (46).

Esta revisão apresentou limitações quanto aos idiomas dos estudos. As buscas foram limitadas aos bancos de dados mencionados na metodologia, não tendo sido realizada busca manual de estudos em outros bancos para complementar a estratégia. A análise do risco de viés apontou para a necessidade de melhorar a qualidade e o rigor das pesquisas nesta temática.

\section{Conclusão}

A avaliação da combinação de tarefas de enfermeiros com outros profissionais e, portanto, da expansão do papel do enfermeiro e da prática colaborativa no primeiro nível da APS mostrou-se efetiva e real em vários países.

A expansão de tarefas, das funções ou do papel do enfermeiro é uma estratégia importante a ser utilizada na superação dos obstáculos impostos pela escassez de RHS e sua má distribuição no provimento de cuidados na APS.

A participação do enfermeiro, combinando suas tarefas com as de outro profissional e, portanto, expandindo seu papel tradicional, pode transformar a sua função, valorizando-a, melhorando a provisão do cuidado, aumentando a adesão e permanência de pacientes na terapêutica prescrita e contribuindo para uma melhor utilização dos RHS e dos custos e melhorando os resultados em saúde. As evidências compiladas e discutidas neste estudo indicam a importância das discussões de políticas para a combinação de tarefas nas equipes de saúde, estruturadas a partir dos princípios da educação e das práticas interprofissionais, e da utilização da EPA na prestação de cuidados na APS.

No ano de 2020, em que o mundo discute a valorização dos profissionais de enfermagem e os aplaude diariamente frente aos seus serviços com pacientes com COVID-19, este estudo é uma evidência aos países sobre o investimento necessário, discussão e incentivo à educação e ao papel do enfermeiro, a EIP e a prática colaborativa como estratégias para o estabelecimento de equipes interprofissionais eficazes e resolutivas .

Investimentos na educação, na regulação e no mercado de trabalho do EPA atuando em atividades combinadas ou interprofissionais, com outros membros da equipe, podem, a curto e médio prazos, determinar e transformar as condições atuais da situação de saúde das populações e melhorar a resolutividade e qualidade dos serviços de saúde na Região das Américas.

Contribuições dos autores. SHDBC e MNFF conceberam a ideia original, planejaram os experimentos, coletaram, analisaram, contribuíram com dados e ferramentas de análise, interpretaram os resultados e escreveram. LR planejou os experimentos, contribuiu com dados de análise e interpretação dos resultados. JRFF e FAMS interpretaram os resultados. Todos revisaram e aprovaram a versão final. 
Conflitos de interesses. Declaramos que não existe qualquer conflito de interesses, como laços financeiros, compromissos acadêmicos, relacionamentos pessoais, crenças políticas ou religiosas e afiliação institucional, entre outros.
Declaração. Os autores são os únicos responsáveis pelas opiniões expressas no manuscrito, que podem não refletir necessariamente a opinião ou política da RPSP / PAJPH e/ou OPAS.

\section{REFERÊNCIAS}

1. Nações Unidas Brasil. 17 Objetivos para transformar nosso mundo | ONU Brasil. Nações Unidas Brasil; 2015. Disponível em: https://nacoesunidas.org/pos2015/ [acessado em 2020 Mai 31]

2. O'Neill K, Takane M, Sheffel A, Abou-Zahr C, Boerma T. Monitoring service delivery for universal health coverage: the Service Availability and Readiness Assessment. Bull World Health Organ. 2013;91(12):923-31. Disponível em: http:/ /www.ncbi.nlm.nih.gov/ pubmed / 24347731 [acessado em 2019 Jul 13]

3. Macaia D, Lapão LV. The current situation of human resources for health in the province of Cabinda in Angola: Is it a limitation to provide universal access to healthcare? Hum Resour Health. 2017;15(1):88. Disponível em: http://www.ncbi.nlm.nih.gov/pubmed / 29282067 [acessado em 2019 Jul 13]

4. Kruk ME, Pate M, Mullan Z. Introducing The Lancet Global Health Commission on High-Quality Health Systems in the SDG Era. Lancet Glob Health. 2017;5(5):e480-1. Disponível em: https://www. thelancet.com/journals/langlo/article/PIIS2214-109X(17)30101-8/ fulltext [acessado em 2020 Jan 6]

5. Pan American Health Organization. PAHO/WHO 29th Pan American Sanitary Conference 2017. Disponível em: https://www.paho.org/ hq/index.php?option=com_content\&view=article\&id=13497:29thpan-american-sanitary-conference\&Itemid=2105\&lang=en [acessado em 2019 Dec 16]

6. Tegegne TK, Chojenta C, Loxton D, Smith R, Kibret KT. The impact of geographic access on institutional delivery care use in low and middle-income countries: Systematic review and meta-analysis. PLoS One. 2018;13(8):e0203130. Disponível em: http://dx.plos. org/10.1371/journal.pone.0203130 [acessado em 2019 Jul 13]

7. World Health Organization. State of the World's Nursing 2020: investing in education, jobs and leadership; 2020.

8. Buchan J, Dal Poz MR. Skill mix in the health care workforce: Reviewing the evidence. Bull World Health Organ. 2002;80(7):57580. Disponível em: https://www.scielosp.org/article/bwho/2002. v80n7/575-580/en/ [acessado em 2020 May 25]

9. Pan American Health Organization. Perspectives and Contributions of Nursing to the Promotion of Universal Health 2020. Disponível em: https://iris.paho.org/handle/10665.2/52121 [acessado em 2020 Mai 31]

10. Cunningham J, O'Toole T, White M, Wells JSG. Conceptualizing skill mix in nursing and health care: An analysis. J Nurs Manag. 2019;27(2):256-63. Disponível em: http://doi.wiley.com/10.1111/ jonm.12673 [acessado em 2019 Jul 13]

11. Sørensen M, Stenberg U, Garnweidner-Holme L. A Scoping Review of Facilitators of Multi-Professional Collaboration in Primary Care. Int J Integr Care. 2018;18(3):13. Disponível em: http:/ /www.ncbi. nlm.nih.gov/pubmed/30220896 [acessado em 2019 Jul 13]

12. World Health Organization. 2020 Vision - The Year of the Nurse and Midwife. Vol. 66, International Nursing Review. Disponível em: https://www.who.int/campaigns/year-of-the-nurse-and-themidwife-2020 [acessado em 2020 Jan 6]

13. Moher D, Shamseer L, Clarke M, Ghersi D, Liberati A, Petticrew $\mathrm{M}$, et al. Preferred reporting items for systematic review and meta-analysis protocols (PRISMA-P) 2015 statement. Syst Rev. 2015;4(1):148-60. Disponível em: https://systematicreviewsjournal.biomedcentral.com/articles/10.1186/2046-4053-4-1 [acessado em 2020 Mai 31]

14. Santos CMDC, Pimenta CADM, Nobre MRC. A estratégia PICO para a construção da pergunta de pesquisa e busca de evidências. Rev Lat Am Enfermagem. 2007;15(3):508-11. Disponível em: http:/ / www.scielo.br/scielo.php?pid=S0104-11692007000300023\&script=sci arttext\&tlng=pt [acessado em 2020 Jan 6]

15. Labrague LJ, McEnroe-Petitte DM, Tsaras K. Predictors and outcomes of nurse professional autonomy: A cross-sectional study. Int
J Nurs Pract. 2019;25(1):e12711. Disponível em: http://doi.wiley. com/10.1111/ijn.12711 [acessado em 2019 Jul 14]

16. Ouzzani M, Hammady H, Fedorowicz Z, Elmagarmid A. Rayyan-a web and mobile app for systematic reviews. Syst Rev. 2016;5(1):210. Disponível em: http://systematicreviewsjournal.biomedcentral. com/articles/10.1186/s13643-016-0384-4 [acessado em 2019 Dec 22]

17. Collaboration Cochrane. EPOC Taxonomy | Cochrane Effective Practice and Organisation of Care 2015. Disponível em: https:// epoc.cochrane.org/epoc-taxonomy [acessado em 2020 May 31]

18. Guyatt G, Oxman AD, Akl EA, Kunz R, Vist G, Brozek J, et al. GRADE guidelines: 1. Introduction - GRADE evidence profiles and summary of findings tables. J Clin Epidemiol. 2011;64(4):383-94. Disponível em: https:/ /www.jclinepi.com/article/S0895-4356(10)00330-6/fulltext [acessado em 2020 Mai 31]

19. Cochrane. EPOC resources for review authors | Cochrane Effective Practice and Organisation of Care 2017. Disponível em: https:// epoc.cochrane.org/resources/epoc-resources-review-authors [acessado em 2020 Jan 8]

20. Anderson RA, Hsieh PC, Su HF. Resource Allocation and Resident Outcomes in Nursing Homes: Comparisons between the Best and Worst. Res Nurs Heal. 1998;21(4):297-313. Disponível em: http:/ / www.ncbi.nlm.nih.gov/pubmed / 9679807 [acessado em 2019 Dec 17]

21. Huba GJ, Cherin DA, Melchior LA. Retention of clients in service under two models of home health care for HIV / AIDS. Home Health Care Serv Q. 1999;17(3):17-26.

22. Kim $\mathrm{H}$, Harrington $\mathrm{C}$, Greene $\mathrm{WH}$. Registered nurse staffing mix and quality of care in nursing homes: A longitudinal analysis. Gerontologist. 2009;49(1):81-90.

23. Shin JH, Park T, Huh I soo. Nursing Staffing and Quality of Life in Western New York Nursing Homes. West J Nurs Res. 2014;36(6):788-805. Disponível em: https://www.ncbi.nlm.nih gov/pubmed/24258404 [acessado em 2019 Dec 17]

24. Lichtenstein BJ, Reuben DB, Karlamangla AS, Han W, Roth CP, Wenger NS. Effect of physician delegation to other healthcare providers on the quality of care for geriatric conditions. J Am Geriatr Soc. 2015;63(10):2164-70. Disponível em: https://www.ncbi.nlm. nih.gov/pmc/articles/PMC4762652/ [acessado em 2019 Dec 17]

25. Jackson GL, Smith VA, Edelman D, Woolson SL, Hendrix CC, Everett $\mathrm{CM}$, et al. Intermediate diabetes outcomes in patients managed by physicians, nurse practitioners, or physician assistants a cohort study. Ann Intern Med. 2018;169(12):825-35. Disponível em: https://annals.org/aim/article-abstract/2716077/ intermediate-diabetes-outcomes-patients-managed-physiciansnurse-practitioners-physician-assistants [acessado em 2019 Dec 17]

26. Dierick-Van Daele ATM, Steuten LMG, Metsemakers JFM, Derckx EWCC, Spreeuwenberg C, Vrijhoef HJM. Economic evaluation of nurse practitioners versus GPs in treating common conditions. Br J Gen Pract. 2010;60(570):28-33. Disponível em: https://www. ncbi.nlm.nih.gov/pmc/articles/PMC2801803/ [acessado em 2020 Mai 31]

27. Van Der Biezen M, Adang E, Van Der Burgt R, Wensing M, Laurant $\mathrm{M}$. The impact of substituting general practitioners with nurse practitioners on resource use, production and health-care costs during out-of-hours: A quasi-experimental study. BMC Fam Pract. 2016;17(1). Disponível em: https://www.ncbi.nlm.nih.gov/pmc/ articles/PMC5020461/ [acessado em 2019 Dec 17]

28. Biezen M Van Der, Wensing M, Burgt R Van Der, Laurant M. Towards an optimal composition of general practitioners and nurse practitioners in out-of-hours primary care teams: A quasi-experimental study. BMJ Open. 2017;7(5). Disponível em: https://bmjopen.bmj. com/content/7/5/e015509 [acessado em 2019 Dec 17]

29. Gerard K, Tinelli M, Latter S, Smith A, Blenkinsopp A. Patients' valuation of the prescribing nurse in primary care: A discrete 
choice experiment. Heal Expect. 2015;18(6):2223-35. Disponível em: https://www.ncbi.nlm.nih.gov/pubmed/24720861 [acessado em 2019 Dec 17]

30. Holt TA, Fletcher E, Warren F, Richards S, Calitri R, Green C, et al. Telephone triage systems in UK general practice: Analysis of consultation duration during the index day in a pragmatic randomised controlled trial. Br J Gen Pract. 2016;66(644):e214-8. Disponível em: https://www.ncbi.nlm.nih.gov/pubmed/26917660 [acessado em 2019 Dec 17]

31. Foster N, McIntyre D. Economic evaluation of task-shifting approaches to the dispensing of anti-retroviral therapy. Hum Resour Health. 2012;10(1):32. Disponível em: https://human-resourceshealth.biomedcentral.com/articles/10.1186/1478-4491-10-32 [acessado em 2019 Dec 17]

32. Jeon YH, Luscombe G, Chenoweth L, Stein-Parbury J, Brodaty H, King M, et al. Staff outcomes from the Caring for Aged Dementia Care REsident Study (CADRES): A cluster randomised trial. Int J Nurs Stud. 2012;49(5):508-18.

33. Song $\mathrm{M}$, Song H. Staff mix and nursing home quality by level of case mix in Korea. Geriatr Gerontol Int. 2019;19(5):438-43. Disponível em: https://onlinelibrary.wiley.com/doi/abs/10.1111/ggi.13631 [acessado em 2019 Dec 17]

34. Myles S, Wyke S, Ibbotson T, Macintyre S, McEwen J, Kelly M. Costs and remuneration for cervical screening in general practice in the west of Scotland. J Health Serv Res Policy. 1996;1(4):217-23.

35. Ivers LC, Jerome JG, Cullen KA, Lambert W, Celletti F, Samb B. Task-shifting in HIV care: A case study of nurse-centered communitybased care in rural Haiti. PLoS One. 2011;6(5).

36. Naruse T, Taguchi A, Kuwahara Y, Nagata S, Sakai M, Watai I, et al. The effect of skill mix in non-nursing assistants on work engagements among home visiting nurses in Japan. J Nurs Manag. 2015;23(4):532-41. Disponível em: https:/ /www.ncbi.nlm.nih.gov/ pmc/articles/PMC5020461/ [acessado em 2019 Dec 17]

37. Maier CB, Batenburg R, Birch S, Zander B, Elliott R, Busse R. Health workforce planning: which countries include nurse practitioners and physician assistants and to what effect? Health Policy (New York). 2018;122(10):1085-92. Disponível em: https://www.ncbi. nlm.nih.gov/pubmed/30241796 [acessado em 2019 Dec 17]

38. Fulton BD, Scheffler RM, Sparkes SP, Auh EY, Vujicic M, Soucat A. Health workforce skill mix and task shifting in low income countries: A review of recent evidence. Vol. 9, Human Resources for Health; 2011. Disponível em: https://human-resources-health.biomedcentral.com/articles/10.1186/1478-4491-9-1 [acessado em 2019 Dec 16]

39. Organização Pan-Americana da Saúde. Diretriz estratégica para a enfermagem na Região das Américas; 2019.
40. Cassiani S, Hoyos MC, Barreto M, Sives K, da Silva FA. Distribución de la fuerza de trabajo en enfermería en la Región de las Américas. Rev Panam Salud Pública. 2018;42:e72. Disponível em: https:/ / doi. org/10.26633/RPSP.2018.72 [acessado em 2019 Dec 16]

41. Lutfiyya MN, Chang LF, McGrath C, Dana C, Lipsky MS. The state of the science of interprofessional collaborative practice: A scoping review of the patient health-related outcomes based literature published between 2010 and 2018. PLoS One. 2019;14(6). Disponível em: https://doi.org/10.1371/journal.pone.0218578

42. Xue Y, Smith JA, Spetz J. Primary Care Nurse Practitioners and Physicians in Low-Income and Rural Areas, 2010-2016. JAMA. 2019;321(1):102-5. Disponível em: https://jamanetwork.com/journals/jama/fullarticle/2720014 [acessado em 2019 Dec 16]

43. Pan American Health Organization. Ampliação do Papel dos Enfermeiros na APS. OPAS; 2018. Disponível em: http://iris.paho.org/ xmlui/handle/123456789/34960 [acessado em 2020 Jan 9]

44. Richardson G, Maynard A. Fewer doctors? More nurses? A review of the knowledge base of doctor-nurse substitution. Working Papers. Centre for Health Economics, University of York; 1995. Disponível em: https://ideas.repec.org/cgi-bin/ htsearch?cmd=Search! \&db=01/01/1990\&de $=\& d t=$ range \&fmt $=$ lon$\mathrm{g} \& \mathrm{~m}=$ all $\& \mathrm{np}=13 \& \mathrm{ps}=50 \& \mathrm{q}=($ saving $++\% 7 \mathrm{C}+$ micro-saving $+\% 7 \mathrm{C}-$ microsaving $+\% 7 \mathrm{C}+$ Aflatoun $+\% 7 \mathrm{C}+$ YouthSafe +$)+++($ "randomize$\mathrm{d}+$ control $^{*}+$ trial $^{\prime}+\% 7 \mathrm{C}++$ "randomised+cont [acessado em 2019 Dec 16]

45. Wind AE, Brage S, Latil F, Williams N. Transfer of tasks in work disability assessments in European social security. Eur J Soc Secur. 2020;22(1):24-38.

46. Freire Filho JR, García JF, De Bortoli Cassiani SH, Menezes da Silva F. Interprofessional Health Education Networks in Latin America and the Caribbean: situation analsys and implementation plans in 19 countries. Soc Innov J. 2019;55(55):1-11. Disponível em: https:// socialinnovationsjournal.org/editions/issue-56/75-disruptiveinnovations / 2984-interprofessional-health-education-networksin-latin-america-and-the-caribbean-situation-analysis-andimplementation-plans-in-19-countries [acessado em 2020 Mai 31]

Manuscrito recebido em 15 de janeiro de 2020. Aceito em versão revisada em 11 de junho de 2020 . 


\section{Skill mix of nurses and primary health care professionals: a systematic review}

ABSTRACT Objective. To evaluate the effectiveness of the strategy of skill mix of nurses and other health care professionals in primary health care.

Methods. Systematic review of the literature aligned with the recommendations of the Preferred Reporting Items for Systematic Reviews and Meta-Analyses (PRISMA), including observational and intervention studies. No restrictions were established for period or country of publication; studies published in Spanish, English and Portuguese were included. The search was carried out in MEDLINE, CINAHL, LILACS, EMBASE and Web of Science electronic databases.

Results. Eighteen studies were analyzed, including 6 from the United States; 3 from the Netherlands; 2 from the United Kingdom; 1 each from Australia, South Africa, South Korea, Scotland, Haiti and Japan; and 1 study with a prospective observational design from 8 countries. The studies reported that the skill mix among health professionals enables a better use of material resources; optimizes time spent on care; improves adhesion to treatment, quality of care and commitment and satisfaction with the work; and reduces the level of psychological distress in nursing professionals.

Conclusions. The expansion of tasks of nurses and other health care professionals, and the skill mix as well as the development of interprofessional teams, are strategies that help to face a scarcity and poor distribution of human resources in urban and non-urban areas with improvement of the health care of the population and satisfaction of professionals and clients.

Keywords $\quad$ Primary health care; nursing, team; health workforce; workforce; Americas. 